\title{
FORECASTING AN EMERGENT SYSTEMIC TREND IN NEUROPHARMACOLOGY
}

\author{
DORU GEORG MARGINEANUa
}

\begin{abstract}
Neuropharmacology (NP), which evolved along with scientific medicine, got treatments for several common disorders of the nervous system. But, most of the NP drugs are just symptomatic, reducing only the manifestations of brain pathologies. Also, for main neuro-psychiatric pathologies (e.g. the neurodegenerative diseases) there is very poor or no medication, and the output of NP drug discovery declined in the last decades. This review paper argues that those drawbacks derive from the reductionist NP leaning towards single-target selective drugs, at odds with the essential complexity of brain functioning and the multifactorial causality of its pathologies. Most active neuro-drugs (e.g. valproate and levetiracetam) are in fact multi-mechanistic since they have been selected by phenotypic screening in vivo, not by single-target binding in vitro. A putative solution of the relative NP stagnation seems to come with the emergent systemic approach of network pharmacology. A plausible flowchart of the main stages of a network NP drug discovery endeavor is finally sketched.
\end{abstract}

Keywords: brain complexity, systems biology, network pharmacology, magic bullet, magic shotgun, multi-mechanistic drug, valproate, levetiracetam, multi-potent drug, poly-therapy

\section{INTRODUCTION}

Neuropharmacology (NP) is an important branch of neuroscience and a primary component of pharma industry, devoted to $i$ ) the study of neurochemical interactions in the central nervous system (CNS), of druginduced changes in nervous system functioning, and to ii) the creation of drugs acting on the nervous system. Its chief importance in both scientific and societal terms derives from the truly foremost role of the brain for us, humans $(H$. sapiens sapiens), more than for any other vertebrate species, and from the high incidence of the neuro- and psycho-pathologies, draining huge

a Department of Neurosciences, Faculty of Medicine and Pharmacy, University of Mons, 7000 Mons, Belgium, Doru.Margineanu@skynet.be 
public costs. CNS diseases are largely recognized as the health challenge of the $21^{\text {st }}$ century, at least $10 \%$ of the global population being now affected by a mental health disorder - a proportion set to increase [1].

NP emerged in the $19^{\text {th }}$ century, along with the scientific medicine and got some major achievements even at that distant times. Among the early feats were the gaseous general anesthesia and the local anesthesia with a synthetic cocaine-like compound (the procaine) devoid of abuse potential. Moreover, NP can rightly boast an impressive series of main achievements, got in the $20^{\text {th }}$ century. Among these are the synthesis and introduction in medical practice of a whole panoply of anti-convulsing drugs, of the typical antipsychotics that treat schizophrenia and severe mania, of the most successful class of benzodiazepine compounds (with sedative, hypnotic, anxiolytic and anti-convulsing effects), and of several classes of antidepressant drugs (from the tricyclic imipramine to the selective serotonin reuptake inhibitors) - to quote just a few major examples [2], [3].

In spite of these, one has however to notice that NP is still in its infancy, as most of the NP drugs are merely symptomatic, not curative, they acting on neurological and psychiatric manifestations, not on the underlying causes of the respective pathologies, which remain as yet largely obscure. Also, NP does not meet several bad medical needs, some common neuropsychiatric pathologies being only poorly treated, if at all. Such are the stroke, the brain and spinal cord trauma, and notoriously the neurodegenerative diseases, chiefly Alzheimer's, whose prevalence rapidly increases in the aging affluent world. Moreover, noteworthy successes of NP often emerged from serendipitous clinical or laboratory observations [4], rather than from attaining purposeful goals of drug discovery programs. Even worse, a decline in output of CNS drug discovery was clearly manifest in the last decades [2], [5] and many of the newly launched medicines were only variations of previous drugs.

This short review, which is an opinion paper, points as a leading cause of the relative lack of efficiency of NP, its unbalanced reductionist leaning, at odds with the intrinsic complexity of CNS functioning. A putative solution brought about by a novel systemic approach is further sketched.

\section{RESULTS AND DISCUSSION}

\section{Basic consequences of the intrinsic complexity of human brain}

Ensuring optimal integration in the milieu and monitoring/coordinating all body functions is an exquisite and demanding communicational activity that led the human brain to emerge as a most intricate web of $>2 \times 10^{10}$ neurons 
and $>10^{11}$ glial cells, each neuron acting as a full-fledged computer, connected with some $10^{4}$ other neurons, and functioning on electrochemical and chemical bases. The unanimously recognized complexity of the brain is approached with concepts from mathematics, physics and computer science [6], [7], [8].

Human brain's amazing organizational intricacy goes along with an oddly intense metabolism, draining over $20 \%$ of the whole body resting energy consumption [9], though the brain accounts for only $2 \%$ of body weight. Its special features render the brain prone to various ailments - physicochemical injury, degeneration, excitability troubles, and intercellular communication alterations - that largely arise from the disruption of milieu's homeostasis needed for suitable neuronal function.

Likewise, brain's sophistication entails the complexity, heterogeneity and, occasionally, the dynamic character of neurological [10] and psychiatric [11] diseases, poorly mastered as yet, making that the etiologies of brainspecific pathologies are but insufficiently understood. This has the corollary that current NP medications are merely symptomatic (not curative). It thus appears fairly obvious that, for achieving to actually cure brain pathologies, the NP should rely on approaches aimed at embracing their intrinsic complexity, transcending the obsessional reductionist search of purportedly highly "selective" drugs, that would strongly interact with just one molecular target, deemed relevant.

The end of last century witnessed the emergence of a far-reaching conceptual move, the ascent of systems biology (SB) [12], [13] that rise above the mere analytic (i.e. reductionist) exclusive focus on the molecular components and highlights the complex interactions within the organisms. Therefore, the pathology is seen to reflect alterations of a network of interactions that underlie each organismic function and the corresponding systems (or network) pharmacology is aimed at normalizing that network.

This novel view on the diseases contests the benefit of a specific interaction of the drug with a unique molecular receptor and its corollary for drug discovery, of aiming to create single-target drugs acting as "magic bullets" (an inspired metaphor introduced by Paul Ehrlich in antimicrobial pharmacology). Network pharmacology searches to act on biological networks rather than on a single molecular target, via either multi-drug therapeutics (poly-pharmacology) or multi-targeted single drugs, appearing as a realistic alternative [14], [15]. The neat truth that drugs that interact with several targets are the most active to treat complex pathologies, such as all the common CNS disorders, led to the proposal that the drugs nonselective for a single target but that selectively interact with several targets involved in a given pathology be dubbed - by a rewording of Ehrlich's metaphor - "magic shotgun" drugs [16]. Such drugs make effective medications for psychiatric pathologies, and the same was proven valid for epilepsy, too [10], [17], [18]. These points are charted in Table 1. 
Table 1. Main focuses of the reductionist (molecule-centric) and the systemic (network-centric) approaches in biology, pathology, and pharmacology, underlying distinct strategies in NP drug discovery.

\begin{tabular}{|l|l|l|}
\hline AREA & Reductionist approach & Systemic approach \\
\hline Biology (basic) & $\begin{array}{l}\text { Molecular-level components \& } \\
\text { specific interactions }\end{array}$ & $\begin{array}{l}\text { Emergent functions in } \\
\text { the integral living system }\end{array}$ \\
\hline Pathology (general) & $\begin{array}{l}\text { Altered elementary } \\
\text { components }\end{array}$ & $\begin{array}{l}\text { Disturbed network of } \\
\text { interactions }\end{array}$ \\
\hline $\begin{array}{l}\text { Pharmacology } \\
\text { (general) }\end{array}$ & $\begin{array}{l}\text { Specific Ligand-Receptor } \\
\text { interaction of Drug-Target }\end{array}$ & $\begin{array}{l}\text { Correction of disturbed } \\
\text { networks }\end{array}$ \\
\hline NP drug discovery & $\begin{array}{l}\text { Rational drug discovery of } \\
\text { clean drugs } \\
\text { Ideal aim }\end{array}$ & Multi-potent therapies bullets \\
\hline
\end{tabular}

Reductionism led to the acclaimed successes of molecular biology, but it fails to account the integrative biological functions that are emergent properties occurring only at organismic level. Likewise, the reductionist goal of single target-selective drugs, contrary to substantiating an efficient drug design, entailed a persistent general decrease in discovery of novel drugs, and this drawback appears particularly marked in NP [19].

\section{The major NP drugs have overt, albeit unwanted, multi- mechanistic nature}

The suggestive label "magic shotgun" was coined upon assessing the mechanisms of the drugs for mood disorders and schizophrenia [16], but it is appropriate for virtually all the drugs effective in treating psychiatric and neurological troubles. Hence, along with the anti-psychotic drugs that modulate (at $\mathrm{nM}-\mu \mathrm{M}$ concentrations) the function of fairly numerous ligandgated and voltage-gated ion channels, also the anti-dementia drugs interact with multiple ion channels [20]. The modulation of multiple molecular targets, i.e. the "pharmacological promiscuity" of the major psychiatric drugs is so manifest that strongly suggests that it might impart clinical efficacy.

Most of the current NP drugs, effective for treating neurological and psychiatric troubles interact with multiple targets simply because these drugs have been developed starting from phenotypic observations, largely serendipitous, in integrated in vivo systems, rather than by target-oriented rational (!) drug discovery. The mechanistic multiplicity of many important NP drugs that were serendipitously discovered is illustrated in Figure 1 by the case of valproate, emblematical for the diversity of biochemical and cellular effects exerted by a rather simple $(\mathrm{MW}=144.2)$ molecule. 


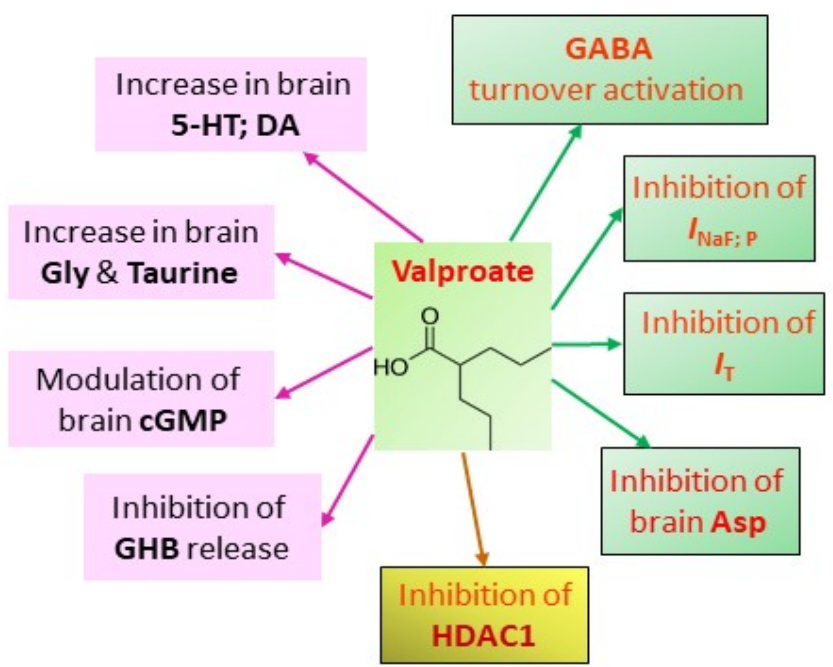

Figure 1. The major NP drug valproate, is largely prescribed as reference antiseizure medication, to treat bipolar disorder and to prevent migraine. Its multiple molecular effects likely contribute to a wide-spectrum anti-seizure activity [21] (green cases at right), mood-stabilizing and anti-migraine activities [22] (pink cases at left), to a possible anti-cancer activity [23] (yellow case at bottom) and to its toxicity. Acronyms: Asp - aspartic acid, cGMP - cyclic guanosine monophosphate, DA - dopamine, GABA - gamma-aminobutyric acid, GHB - gamma-hydroxybutyric acid, Gly - glycine, HDAC1 - histone deacetylase 1, 5-HT - serotonin, INa F; Psodium currents, fast and lasting, $/ T-$ transient calcium current. Adapted from [21].

The epithet "magic shotgun" undoubtedly fits the antipsychotic drugs for schizophrenia and other severe mental disorders since all of them actually have numerous targets, up to 26 for clozapine and quetiapine [24]. However, this equally holds for many other effective NP drugs. Thus, the fact that all the classical (reference) anti-seizure drugs (ASDs) and the more clinically useful new generation ASDs exert their hypo-excitatory effects upon interacting with multiple ion channels was repeatedly argued and detailed [10], [17], [18].

The multiplicity of effects is manifest even in those cases where a welldefined binding site was revealed for a given drug, by a purposeful investigation subsequent to the phenotypic revealing of that drug. Such is the representative case of the new generation ASD levetiracetam (LEV), illustrated in Figure 2. That figure displays only the cellular effects and the molecular interactions of LEV deemed germane for the anti-seizure activity of that remarkable ASD, not all of its numerous reported effects. 


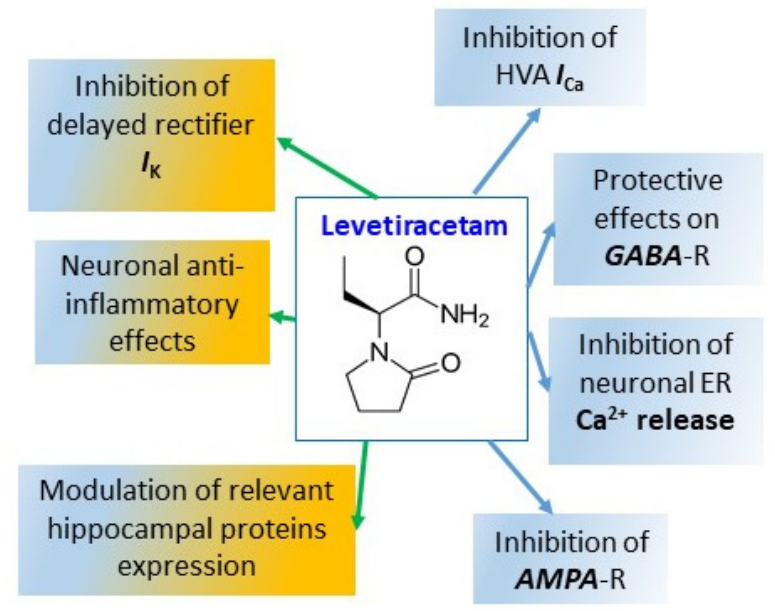

Figure 2. The newer (3 $3^{\text {rd }}$ generation) ASD levetiracetam (LEV) has multiple interactions, generally rather mild and of modulatory type [25] with several types of neuronal ionic channels involved in controlling neuronal excitability: the highvoltage activated (HVA) $\mathrm{Ca}^{2+}$ channels [26], the GABA-activated $\mathrm{Cl}^{-}$channels [27], the AMPA type of glutamate-activated channels [28], and it inhibits the release of $\mathrm{Ca}^{2+}$ from neuronal endoplasmic reticulum (ER) [29]. Beside these discernably anti-seizure effects (right side of the figure), LEV also exerts more intricate excitability-modulating effects, such as inhibition of the delayed rectifier $\mathrm{K}^{+}$ channels [30], modulation of hippocampal protein expression, counteracting inflammatory changes in astroglia etc. (references quoted in [2], from which was adapted this illustration). LEV shows a multi-mechanistic feature shared by all NP drugs revealed by phenotypic screening in vivo, even though a specific binding site has been afterwards identified (for LEV, the synaptic vesicle protein SV2A [31]).

The case of LEV (familiar to the present author) is particularly relevant for the subject of the current review. Launched in 2000 in both USA and Europe, LEV became within a few years the most prescribed ASD of newer generation and the first ASD to rise at the status of blockbuster. Specialized medical reviews have long concluded that LEV is "a safe, broad-spectrum anticonvulsant drug with highly beneficial pharmacokinetic properties, a favorable long-term retention rate, and a high responder rate" [32]. Also, the continual medical and scientific interest of that drug is clearly attested by a PubMed search of 9 July 2021 with the subject "levetiracetam", that indicated 4,484 publications to date, of which 439 appeared solely in the year 2020, and among which there are 844 review papers. 
Chemically, LEV is (S)- $\alpha$-ethyl-2-oxo-1-pyrrolidine-acetamide, i.e. the (S)-enantiomer of the ethyl analog of piracetam (2-oxo-1-pyrrolidineacetamide) - the prototype drug of the widely used, though controversial nootropic products [33], purportedly cognitive enhancers. It arose from a chemical synthesis program aimed to generate a better cognitive enhancer, which it did not prove to be, but a general preclinical in vivo screening in rodents revealed some less conventional anti-convulsing activity. Professional extension of the early observations in living animals to studies in vitro, revealed a correlation of the anticonvulsant effect with a specific binding on a protein present in the brain. That initially mysterious "LEV-binding site" was identified, after nearly a decade, to be the synaptic vesicle protein SV2A [31].

Even before that delayed identification, the correlation between the anticonvulsant activity in a suitable screening test in vivo and the affinity towards the LEV-binding site of the different racetam compounds (sharing the pyrrolidone nucleus of piracetam [34]) became a useful tool allowing a target-oriented drug-discovery. It resulted in the selection of a successor of levetiracetam, its 4- $n$-propyl analogue - the brivaracetam (BRV) - that has binding affinity and anticonvulsant potency about 10 -fold greater than LEV in animal tests [35]. In 2016, BRV became a marketed anticonvulsant drug.

The following Figure 3 shows that the affinity for SV2A of racetam compounds is more manifest when the molecules are bigger, getting a structure that likely fits better the binding site on the receptor. Thus, piracetam has no noticeable SV2A affinity, while LEV (its (S)- $\alpha$-ethyl derivative) has a significant affinity and BRV (the 4- $n$-propyl derivative of LEV) gets a further increased affinity. That simple plot substantiates two worthy remarks. Firstly, it illustrates in a relevant particular case the general non-specificity of piracetam, a small molecule whose nearly negligible interaction with all chemical components of the organism might probably explain its unusual lack of toxicity [36], but which also obscures its purported effects. However, several other small molecules exert non-specific effects on the brain, being either depressant (e.g. the anesthetic gases) or stimulant (e.g. the methylxanthines). A most prominent example of a small molecule non-specific CNS drug is the ethanol (MW $=46.1)$ that has multiple effects on the brain functions, not all of them accounted by obvious Ligand-Receptor interactions.

Another remark is the practical utility for drug discovery of identifying a specific binding in vitro, provided that the affinity of that binding be tightly correlated with a functional effect in vivo. That correlation supports an efficient selection from chemical libraries of candidate molecules to optimize a confirmed therapeutic activity, i.e. to find worthy successors of existing drugs. Obviously, however, those successors will be of basically the same type as the parent drug. 


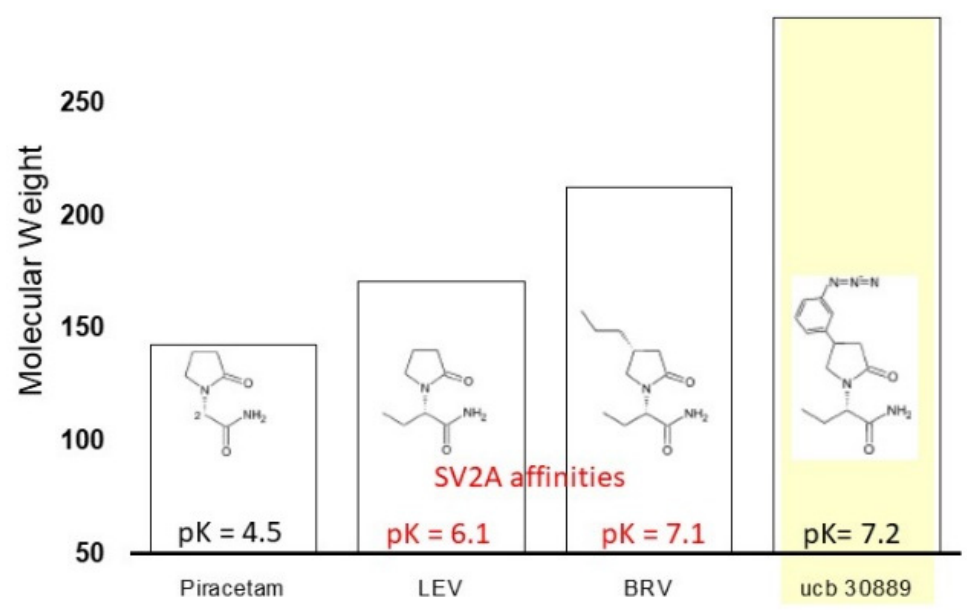

Figure 3. Molecular weight (height of the columns) of four racetam compounds the nootropic drug piracetam, the ASDs levetiracetam (LEV) and brivaracetam (BRV), and a derivative of LEV (ucb 30889) used as experimental marker - having increasing affinities (the pK values given on each column) for the protein SV2A. Drawing based on crude data from [31], [37], [38].

The account of the anti-convulsive properties of the higher-affinity SV2A ligand BRV [35] was editorially hailed in the British Journal of Pharmacology as "a rational drug discovery success story" [39]. However, a truly stern reality concerning all the current ASDs, including those recently launched, is that none of them actually cures epilepsy, but only reduce its symptoms. Epilepsy is among the most common chronic neurological pathologies, often life-threating, affecting up to $1 \%$ of world population and which was mentioned in writings since more than four thousand years. Its omnipresence in human history entailed considerable focus of NP, making that more than 20 ASD are currently available, a dozen of them licensed in this century. But, in spite of the profusion of ASD options, about $30 \%$ of epileptic patients remain drug-refractory [40] and - most disturbingly - the proportion of patients with drug-refractory seizures did not decline since more than half a century [41].

Comprehensibly, drug resistance in epilepsy is the subject of sustained scientific attention [40], [41], [42], [43], but a worrying lack to meet tough medical needs is in no way limited to anti-epileptic pharmacology, it being inherent to virtually all the domains of NP, as briefly outlined in the Introduction. Because a leading cause of NP deficits likely relies on its insufficient matching 
of the unequaled intrinsic complexity of human brain, a conceivable solution might be brought by the emerging systemic approach of network pharmacology, as noted in the above section on Basic consequences of the intrinsic complexity of human brain.

The idea that, for drugs intended to treat complex pathologies, adjusting the mismatch between multiple receptors is more important than just fixing the anomaly of a single one - so that absolute selectivity is not a suitable ideal in NP - was occasionally expressed prior to the advent of systems pharmacology (for discussion see [21]). But, the preference for single-target selectivity prevailed in NP drug discovery, stirred by the reductionism of molecular biology, prior to the current "postgenomic era". Along with the reductionist mindset, the selectivity dream was also supported by a marketing-driven wish that a medicine should have a forthright image, believed easier to convey to prescribers [18].

Within the integrative SB view, a given CNS pathology reveals a disturbed network of interactions, so that the network NP aims at returning to normal that pathology-disturbed network, via a multi-component therapy or multi-potent drugs. Thus, a first, though not foremost, objective of the systemic approach of NP is a rational poly-therapy, upon identifying combinations of existing neuro-drugs (and adjuvants) with efficacy optimized for each patient. Poly-therapy of CNS pathologies was empirically pursued before the advent of network pharmacology, but the SB approach would bestow on multi-drug therapy a real rationality.

On the other hand, the main objective of network NP should be the design of novel innovative drugs appropriately acting on the ensemble of molecular entities critically involved in each well-defined neuro- / psychopathology, to correct the respective disturbed network of molecular interactions. Basically, the application of SB to drug discovery in general involves several types of activities [44]:

- integration of 'omics' data sets and of information available in the literature on responses at cell and organ levels,

- computer modeling of the disease patho-physiology and in silico screening,

- experimental approaches to record disease-relevant biological responses by using complex human cell-based assays and occasionally animal models, to capture emergent properties.

Tentatively, the main steps of plausible network NP endeavors can be visualized as in the following Figure 4. 


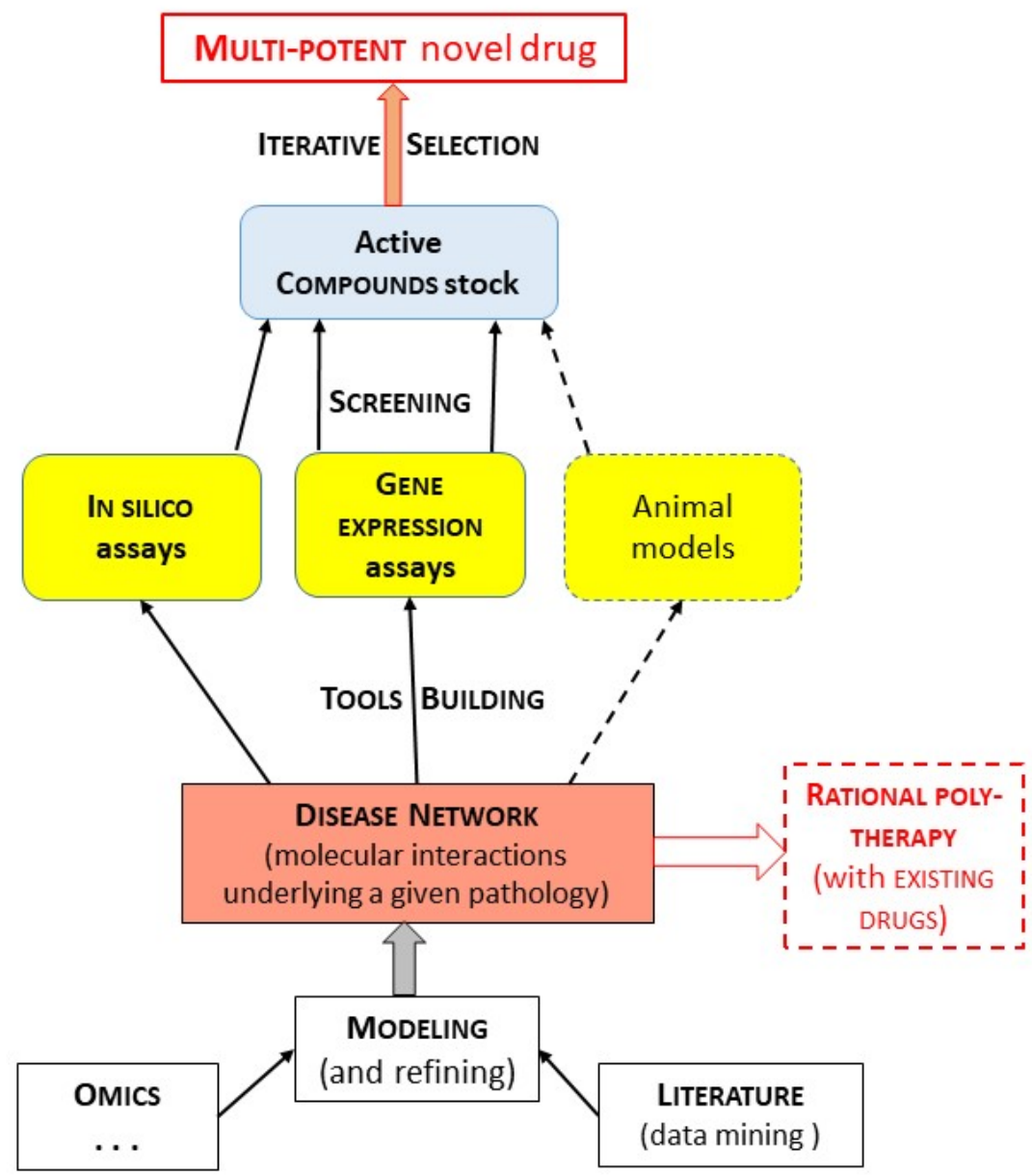

Figure 4. Putative succession of main stages of a network NP drug discovery process aimed at medications for neuro- / psycho- pathologies. Revealing the network of molecular interactions that underlie that pathology in a representative homogeneous group of patients, characterized by the omics (the disease network) sets a rational basis for poly-therapy with possibly existing drugs. It mainly provides the background for creating screening tools to select from chemical libraries active compounds that might finally permit to develop multi-potent drugs. This scheme, adapted from [43], is meant just to illustrate a conceivable endeavor. 
Attaining the goal of multi-potent (neuro-) drugs is an arduous task, since optimizing all at once multiple desired activities of a compound, with simultaneous control of unwanted effects, is by far more intricate and cumbersome than the single-target strategy. Yet, significant bioinformatics and chemo-informatics resources are already set in place, computational methods have been put forward, and all advance rapidly.

A wealth of relevant accounts of efforts and achievements advancing the NP along the systemic network pharmacology continuously appear. They refer to quite various aspects, such as: systems level analysis of brain disease-associated proteome alterations [45], network pathophysiology of particular CNS disorders [46], computational approaches for improving NP and innovative drug discovery [47], [48], neuroprotective drugs revealed using artificial intelligence [49], status, opportunities, and challenges of quantitative systems NP [50].

These are but some examples, which, however, illustrate the sustained progress of systemic, network-centric approaches in NP.

\section{CONCLUSIONS}

The high scientific and societal importance of NP arises from the unique role of the human brain and the high incidence of the neuro- and psycho-pathologies. NP got some major medical achievements, such as anesthesia and the creation of a large panoply of key drugs to alleviate neurological and psychiatric diseases: many anti-convulsing drugs, the antipsychotics, the benzodiazepine compounds (sedative, hypnotic, anxiolytic, anti-convulsing), and several classes of antidepressant drugs.

But, most of the NP drugs are only symptomatic, relieving the manifestations, not curing the causes of the respective pathologies, which are still vaguely understood. Also, some common neuro-psychiatric pathologies (stroke, CNS trauma and neurodegenerative diseases) are only poorly or not at all treated. Besides, the invention of innovative neuro-drugs clearly declined in the last decades. A chief source of these drawbacks is the mismatch between the unparalleled intrinsic complexity of human brain, making that CNS pathologies are essentially multifactorial, and the unduly reductionist NP orientation towards single-target selective drugs. Combined with a distorted obsession of economic efficacy, the reductionism led NP to rely excessively on the screening in vitro, leaving aside the phenotypic screening in vivo.

But, the ascent of SB, in the postgenomic era, revealed that pathologies are alterations of the network of interactions that underlie each organismic function, so that a novel network pharmacology aims normalizing networks. 
Hence, instead of single-target brain drugs, network NP would overtly search multi-potent drugs. Actually, the best existing neuro-drugs are multi-mechanistic, just because they have been discovered (often serendipitously) by phenotypic screening. The major drug valproate clearly shows manifold actions on various molecular targets in the neurons. Even neuro-drugs for which a specific binding site has been discovered, after their development via in vivo tests, are nevertheless multi-mechanistic. Such is the case of the major new generation ASD levetiracetam.

The purposeful invention of multi-potent drugs has to integrate omics and literature data on responses at cell and organ levels, computer modeling of the disease pathophysiology, in silico screening, and the record of diseaserelevant biological responses in human cell-based assays. The task is highly demanding, but some tools are already in place and they progress rapidly.

\section{DEDICATION}

This paper is devoted to the memory of my faithful friend of long date Prof. Dr. Petre T. Frangopol, Honor Member of the Romanian Academy.

\section{REFERENCES}

1. A. G. Phillips; P. Hongaard-Andersen; R. A. Moscicki; B. Sahakian; R. Quirion; K. R. R. Krishnan; T. Race; Int. J. Neuropsychopharmacol., 2015, 19, 1-16

2. D. G. Margineanu; BioSystems, 2016, 141, 1-9

3. E. Shorter; Curr. Opin. Psychiatry, 2008, 21, 593-597

4. T. A. Ban; Dialogues Clin. Neurosci., 2006, 8, 335-344

5. B. H. Yokley; M. Hartman; B. S. Slusher; ACS Chem. Neurosci., 2017, 8, 429431

6. W. Singer; EMBO Rep., 2007, 8, S16-S19

7. Q. Mu; Y. Chen; J. Wang; Genomics Proteomics Bioinformatics, 2019, 17, 344-366

8. J. L. Ji; M. Spronk; K. Kulkarni; G. Repovš; A. Anticevic; M. W. Cole; Neuroimage, 2019, 185, 35-57

9. S. B. Laughlin; T. J. Sejnowski; Science, 2003, 301, 1870-1874

10. D. G. Margineanu; Epilepsy Res., 2012, 98, 104-115

11. T. Takahashi; Prog. Neuropsychopharmacol. Biol. Psychiatry, 2013, 45, 257-265

12. H. Kitano; Science, 2002, 295, 1662-1664

13. U. Sauer; M. Heinemann; N. Zamboni; Science, 2007, 316, 550-551

14. C. T. Keith; A. A. Borisy; B. R. Stockwel; Nature Rev. Drug Discov., 2005, 4, 71-78

15. A. L. Hopkins; Nature Chem. Biol., 2008, 4, 682-690 
16. B. L Roth; D. J. Sheffler; W. K., Kroeze; Nature Rev. Drug Discov., 2004, 3, 353-359

17. M. T. Bianchi; J. Pathmanathan; S. S. Cash; Med. Hypotheses, 2009, 72, 297305

18. D. G. Margineanu; Epilepsy Behav., 2014, 38, 131-142

19. J. Arrowsmith; Nature Rev. Drug Discov., 2011, 10, 1

20. M. T. Bianchi; Med. Hypotheses, 2010, 74, 297-300

21. D. G. Margineanu; Systems (network) pharmacology for brain functionality restoration. In Homeostatic Control of Brain Function; D. Boison, S. Masino Eds.; Oxford University Press, New York, USA, 2015; pp. 231-247

22. C.-T. Chiu; Z. Wang; J. G. Hunsberger; D.-M. Chuang; Pharmacol. Rev., 2013, 65, 105-142

23. S. Chateauvieux; F. Morceau; M. Dicato; M. Diederich; J. Biomed. Biotech., 2010, article ID 479364, doi:10.1155/2010/479364

24. J. Sun; H. Xu; Z. Zhao; Chem. Biodiv., 2012, 9, 900-910

25. D. G. Margineanu; H. Klitgaard; Levetiracetam mechanisms of action. In Antiepileptic Drugs, $5^{\text {th }}$ ed.; R. H. Levy, R. H. Mattson, B. S. Meldrum, E. Perucca Eds.; Lippincott Williams \& Wilkins, Philadelphia, USA, 2002; pp. 419-427

26. I. Niespodziany; H. Klitgaard; D.G. Margineanu; Neurosci. Lett., 2001, 306, 5-8

27. P. Poulain P.; D. G. Margineanu; Neuropharmacol., 2002, 42, 346-352

28. I. Carunchio; M. Pieri; M. Ciotti; F. Albo; C. Zona; Epilepsia, 2007, 48, 654-662

29. M. Ängehagen; D. G. Margineanu; E. Ben-Menachem; L. Ronnback; E. Hansson. H. Klitgaard; NeuroReport, 2003, 14, 471-475

30. M. Madeja; D. G. Margineanu; A. Gorji; E. Siep; P. Boerrigter; H. Klitgaard; E.-J. Speckmann; Neuropharmacol., 2003, 45, 661-671

31. B. A. Lynch; N. Lambeng; K. Nocka; P. Kensel-Hammes; S. M. Bajjalieh; A. Matagne; B. Fuks; Proc. Nat. Acad. Sci. USA, 2004, 101, 9861-9866

32. T. De Smedt; R. Raedt; K. Vonck; P. Boon; CNS Drug Reviews, 2007, 13, 5778

33. D. G. Margineanu; Rev. Quest. Sci, 2011, 182, 33-52

34. B. M. Kenda; A. C. Matagne; P.E. Talaga; P. M. Pasau; E. Differding; B. I. Lallemand; A. M. Frycia; F. G. Moureau ; H. V. Klitgaard; M. R. Gillard; B. Fuks; P. Michel; J. Med. Chem., 2004, 47, 530-549

35. A. C. Matagne; D. G. Margineanu; B. Kenda; P. Michel; H. Klitgaard; Br. J. Pharmacol., 2008, 154, 1662-1671

36. B. Winblad; CNS Drug Reviews, 2005, 11, 169-182

37. M. Gillard; B. Fuks; P. Michel; P. Vertongen; R. Massingham; P. Chatelain; Eur. J. Pharmacol., 2003, 478, 1-9

38. P. von Rosenstiel; Neurotherapeutics, 2007, 4, 84-8

39. M. A. Rogawski; Br. J. Pharmacol., 2008,154, 1555-1557

40. A. Golyala; P. Kwan; Seizure, 2017, 44,147-156

41. D. G. Margineanu; H. Klitgaard; Expert Opin. Drug Discov., 2009, 4, 23-32

42. W. Löscher; H. Potschka; S. M. Sisodiya; A. Vezzani; Pharmacol. Rev., 2020, $72,606-638$

43. D. G. Margineanu; Med. Res. Arch., 2021, 9, in press 
44. E. C. Butcher; E. L. Berg; E. Kunkel; Nature Biotechnol., 2004, 22, 1253-1259

45. M. Keck; G. Androsova; F. Gualtieri; A. Walker; E.-L. von Rüden; V. Russmann; C. A. Deeg; S. M. Hauck; R. Krause; H. Potschka; Neurobiol. Dis., 2017, 105, 164-178

46. A. B. Elgoyhen; B. Langguth; S. Vanneste; D. DeRidder; Front. Systems Neurosci., 2012, 6, doi: $10.3389 /$ fnsys.2012.00001

47. K. Shameer; A. Nayarisseri; F. X. R. Duran; H. González-Díaz; Curr. Neuropharmacol., 2017, 15, 1058-1061

48. A. Talevi; Expert Opin. Drug Discov., 2016, 11, 1001-1016

49. D. Romeo-Guitart; J. Forés; M. Herrando-Grabulosa; R. Valls; T. Leiva-Rodríguez; E. Galea; F. González-Pérez; X. Navarro; V. Petegnief; A. Bosch; M. Coma; J. M. Mas; C. Casas; Sci. Rep. 2018, 8,1879 doi:10.1038/s41598-018-19767-3

50. H. Geerts; J. Wikswo; P. H. van der Graaf; J. P.F. Bai; C. Gaiteri; D. Bennett; S. E. Swalley; E. Schuck; R. Kaddurah-Daouk; K. Tsaioun; M. Pelleymounter; CPT Pharmacometrics Syst. Pharmacol., 2020, 9, 5-20 